\title{
CONSERVATION LAWS FOR INCOMPRESSIBLE FLUIDS
}

\author{
G. CAVIGLIA \\ Department of Mathematics \\ Via L.B. Alberti 4 \\ 16132 Genova, Italy \\ A. MORRO \\ Department of Biophysical and Electronic Engineering \\ Viale Causa 13 \\ 16145 Genova, Italy \\ (Received November 18, 1987)
}

\begin{abstract}
By means of a direct approach, a complete set of conservation laws for incompressible fluids is determined. The problem is solved in the material (Lagrangian) description and the results are eventually rewritten in the spatial (Eulerian) formulation. A new infinite family of conservation laws is determined, besides those for linear momentum, angular momentum, energy and helicity.
\end{abstract}

KEY WORDS AND PHRASES. Conservation laws, incompressible fluids, Lagrangian description, complete classification.

1980 AMS CLASSIFICATION CODES. 76C99, 35Q20

\section{INTRODUCTION.}

The determination of conservation laws, and of the corresponding invariants, for the 3dimensional Euler equations can be of fundamental importance in studying existence and uniqueness of solutions [1-3]. In this scheme, Serre examined Euler's equations for the motion for an incompressible inviscid fluid under the assumption that the conserved quantities are independent of the space-time variables. He has shown [1] that the conserved quantites are in fact the linear momentum, energy, and the so-called helicity [4], only. Later, on adopting a Hamiltonian formulation, Olver has derived the conservation law of angular momentum by considering the generators of symmetry transformations [5].

Recently, an analysis of inviscid compressible fluid flows based on infinitesimal generators of symmetry transformations and adjoint variables $[6,7]$ has led to the discovery of an additional conservation law [8] describing, in a sense, the center-of-mass theorem. Taking this result as a motivation and looking for a more exhaustive approach, the problem was dealt with in the material description [9], where the Euler equations allow for a variational formulation and thus Noether's theorem permits (cf. [10]) the determination of a complete set of conservation laws. Really, new conservation laws depending on arbitrary functions, besides the center-of-mass theorem, have been determined, but the procedure is not completely exhaustive, because of some technical limitations that have been imposed from the very beginning, in order to render the required calculations more manageable. 
In this paper we consider the incompressible fluid model and look for the full set of independent conservation laws, without any restrictive assumption on the form of such laws. In this case no variational formulation, and thus none of the various forms of Noether's theorem, is available. That is why we adopt a direct approach and seek solutions to the vanishing of a 4-dimensional divergence. We find it convenient to solve the problem in the material description and, eventually, to rewrite the results in the spatial (Eulerian) one.

As a result of our approach, the complete set of conserved vectors is given and, as a byproduct, a new infinite class of conservation laws is determined explicitly. This class extends to incompressible fluids a remarkable result discovered very recently for compressible fluids [9]. Incidentally, the procedure elaborated in this paper is likely to work for more involved situations.

\section{PRELIMINARIES.}

To set up a general framework for conservation laws in fluid dynamics we consider a system described by $n$ functions $\phi_{\beta}, \beta=1,2, \ldots, n$, of the (space-time) variables $X_{\Sigma}, \Sigma=1,2, \ldots, m$. The functions $\phi_{\beta}$ satisfy the second order system of differential equations

$$
F_{\alpha}\left(X_{\Lambda}, \phi_{\beta}, \phi_{\beta, \Sigma}, \phi_{\beta, \Sigma \Lambda}\right)=0, \quad \alpha=1,2, \ldots, n,
$$

where a comma followed by a letter, $\Sigma$ say, denotes the partial derivative with respect to $X_{\Sigma}$. The functions $F_{\alpha}$ are supposed to be of class $C^{1}$ with respect to their argument.

Let $D_{\Sigma}$ denote the (total) derivative with respect to $X_{\Sigma}$. A conservation law for the system (2.1) is a second order differential equation

$$
D_{\Sigma} I_{\Sigma} \doteq 0
$$

for suitable functions $I_{\Sigma}$ of the form

$$
I_{\Sigma}=I_{\Sigma}\left(X_{\Lambda}, \phi_{\beta}, \phi_{\beta, \Sigma}\right) \text {. }
$$

The symbol $\doteq$ is a reminder that equality is required to hold in connection with the solutions $\phi_{\beta}$ to (2.1) only. Operatively, determining a conservation law amounts to finding a m-tuple of functions $I_{\Sigma}$ such that (2.2) is satisfied identically by the solutions to (2.1).

Trivial conservation laws arise when the $I_{\Sigma}$ 's vanish for all solutions to (2.1) or when (2.2) holds for all functions $\phi_{\beta}$ regardless of whether they solve the system (2.1) [10]. Such trivial conservation laws do not provide any information about the properties of the solutions and then two conservation laws are regarded as equivalent whenever they differ by a trivial conservation law. Non-equivalent conservation laws are said to be independent. Accordingly we are only interested in the determination of independent and non-trivial conservation laws.

\section{GOVERNING EQUATIONS.}

Let $\mathbf{v}$ be the velocity field of the fluid and $p$ the pressure. Moreover let latin indices run over $1,2,3$ and denote Cartesian components. So $x_{i}$ is the $i$-th component of the position vector $\mathbf{x}$ in the three-dimensional Euclidean space $\mathcal{E}^{3}$. The motion of an inviscid, incompressible fluid is governed by the system of differential equations [11]

$$
\frac{\partial v_{i}}{\partial t}+v_{j} \frac{\partial v_{i}}{\partial x_{j}}+\frac{1}{\rho} \frac{\partial p}{\partial x_{i}}=0
$$




$$
\frac{\partial v_{j}}{\partial x_{j}}=0
$$

in the unknown functions $v_{i}, p$. Equations (3.1)-(3.2) reflect the Eulerian description of motion where $v_{i}$ and $p$ are viewed as $C^{1}$ functions of $x \in \mathcal{E}^{3}$ and time $t \in R$.

As it often happens, here it is convenient to account also for the motion of a fluid particle, usually denoted by a function $x(t)$. To avoid any confusion about the meaning of the symbol $x$ (independent variable or function of time) one might adopt the Lagrangian description where the particles are labelled by the position $\mathbf{X}$ they occupy in a reference configuration $\mathcal{F} \in \mathcal{E}^{\mathbf{s}}$. In that case the independent variables are $\mathbf{X} \in \mathcal{F}$ and $t \in R$ and the unknown functions are the motion $\mathbf{x}(\mathbf{X}, t)$ and the pressure $p(\mathbf{X}, t)$. Accordingly, adopting the Lagrangian description in the standard way [11] makes (3.1)-(3.2) in a second order differential system.

For the present purposes the Lagrangian description as such has another drawback, besides that of increasing the order of the system, because it makes less immediate to contrast our results with those obtained by Olver [5] within the Eulerian description. That is why eventually the results determined via the material formulation will be given the corresponding Eulerian form.

For convenience in calculations we denote by upper case indices the Cartesian components of $\mathbf{X}$. Moreover, in connection with the function $\mathbf{x}(\mathbf{X}, t)$ and its inverse $\mathbf{X}(\mathbf{x}, t)$ we let

$$
x_{i H}=\frac{\partial x_{i}}{\partial X_{H}}, \quad x_{i t}=\frac{\partial x_{i}}{\partial t}, \quad X_{H i}=\frac{\partial X_{H}}{\partial x_{i}} .
$$

Similarly,

$$
p_{H}=\frac{\partial p}{\partial X_{H}}, \quad p_{t}=\frac{\partial p}{\partial t} .
$$

Accordingly, the system (3.1)-(3.2) may be written in the form

$$
\begin{gathered}
x_{i t t}+\frac{J}{\rho_{0}} p_{H} X_{H i}=0, \\
x_{i t H} X_{H i}=0,
\end{gathered}
$$

where the condition of mass conservation , $\rho=\rho_{0} / J$, has been used with $J=\operatorname{det}\left(x_{i H}\right)$. Really $J=1$ but, to simplify the comparison with the compressible case, we prefer to write just $J$.

For later convenience we write now some identities that will be freely used without further reference:

$$
J X_{A a}=\frac{\partial J}{\partial x_{a A}}=\frac{1}{2} \epsilon_{a h k} \epsilon_{A H K} x_{h H} x_{k K}, \quad D_{H}\left(J X_{H h}\right)=0
$$

\section{GENERAL FORM OF THE CONSERVATION LAWS.}

Back to the general framework of section 2, we identify the unknown fields $\phi_{\beta}$ with $x_{i}$, $i=1,2,3$, and $p$. The independent variables are $X_{H}, H=1,2,3$, and $t$. Then we search for conservation laws (2.2) in the form

$$
D_{t} I\left(X_{H}, t, x_{i}, p, x_{i t}, x_{i H}\right)+D_{K} I_{K}\left(X_{H}, t, x_{i}, p, x_{\imath t}, x_{i}\right) \doteq 0
$$

where $I$ is the conserved density and $I_{K}$ is the associated flux. As a consequence, the helicity integral $[1,4,5]$ is ruled out, since it involves the curl of the velocity field. In view of the previous discussion on equivalence, it is also to be observed that the density is only defined up to the divergence of a spatial vector; this remark will lead to a simplification in the subsequent calculations. 
The explicit form of (4.1) reads

$$
\begin{aligned}
\frac{\partial I}{\partial t} & +\frac{\partial I}{\partial x_{i}} x_{i t}+\frac{\partial I}{\partial p} p_{t}+\frac{\partial I}{\partial x_{i t}} x_{i t t}+\frac{\partial I}{\partial x_{i H}} x_{i H t} \\
& +\frac{\partial I_{K}}{\partial X_{K}}+\frac{\partial I_{K}}{\partial x_{i}} x_{\imath K}-\frac{\rho_{0}}{J} \frac{\partial I_{K}}{\partial p} x_{v H} x_{t t t}+\frac{\partial I_{K}}{\partial x_{v t}} x_{v t K}+\frac{\partial I_{K}}{\partial x_{i H}} x_{i H K} \doteq 0
\end{aligned}
$$

where (3.3) has been taken into account. Here $p_{t}, x_{i t t}$, and $x_{i H K}$ are regarded as arbitrary quantities while $x_{\imath t H}$ satisfies the constraint equation (3.4). Hence (4.2) holds identically if and only if

$$
\begin{gathered}
\frac{\partial I}{\partial p}=0 \\
\frac{\partial I}{\partial x_{i t}}-\frac{\rho_{0}}{J} \frac{\partial I_{K}}{\partial p} x_{i K}=0 \\
\frac{\partial I_{K}}{\partial x_{i H}}+\frac{\partial I_{H}}{\partial x_{i K}}=0 \\
\frac{\partial I}{\partial t}+\frac{\partial I}{\partial x_{i}} x_{i t}+\frac{\partial I_{K}}{\partial X_{K}}+\frac{\partial I_{K}}{\partial x_{i}} x_{i K}=0 \\
\left(\frac{\partial I}{\partial x_{i K}}+\frac{\partial I_{K}}{\partial x_{i t}}\right) x_{i t K} \doteq 0
\end{gathered}
$$

Once we account for the constraint (3.4), the condition (4.7) yields [10]

$$
\frac{\partial I}{\partial x_{i K}}+\frac{\partial I_{K}}{\partial x_{i t}}-2 J \lambda X_{K i}=0
$$

where $2 J \lambda$ is the Lagrange multiplier and $\lambda$ is allowed to depend on $X_{H}, t, x_{i}, p, x_{t t}$.

The solution to (4.5) may be represented as

$$
I_{K}=\beta_{l} \epsilon_{l r}, \epsilon_{K P Q} x_{r P} x_{\bullet Q}+\omega_{j H K} x_{j H}+\alpha_{K}
$$

where $\beta_{l}, \omega_{j H K}$, and $\alpha_{K}$ are functions of $X_{H}, t, x_{i}, p, x_{i t} ; \omega_{j H K}=-\omega_{j K H}$. Then (4.4) and (4.8) yield respectively

$$
\begin{gathered}
\frac{\partial I}{\partial x_{i t}}=\frac{\rho_{0}}{J}\left(\frac{\partial \beta_{l}}{\partial p} \epsilon_{l r,} \epsilon_{K P Q} x_{r P} x_{\bullet Q}+\frac{\partial \omega_{j H K}}{\partial p} x_{j H}+\frac{\partial \alpha_{K}}{\partial p}\right) x_{i K} \\
\frac{\partial I}{\partial x_{i K}}=2 J\left(\lambda \delta_{i l}-\frac{\partial \beta_{l}}{\partial x_{i t}} J\right) X_{K \imath}-\frac{\partial \omega_{j H K}}{\partial x_{i t}} x_{j H}-\frac{\partial \alpha_{K}}{\partial x_{i t}} .
\end{gathered}
$$

where $\delta_{i l}$ denotes as usual the Kronecker symbol. Eqs.(4.3), (4.10), and (4.11) allow the determination of the explicit dependence of $I$ on $p, x_{i t}$, and $x_{i K}$. For example, since $I$ is independent of $p$ in view of (4.3), it follows in particular from (4.10) that $\beta_{l}$ may be represented as

$$
\beta_{l}=p \hat{B}_{l}+\hat{b}_{l}
$$

with $\hat{B}_{l}$ and $\hat{b}_{l}$ as functions of $X_{H}, t, x_{i}, x_{t}$. Then, after substitution into (4.11), we can use the arbitrariness in $\lambda$ to set $\lambda \delta_{i l}=\partial \hat{B}_{l} / \partial x_{i t}$, so as to eliminate dependence on $p$ in the contribution proportional to $X_{K \iota}$. Back to more general considerations, when we impose the standard integrability conditions on (4.3), (4.10), and (4.11) we find further restrictions on the arbitrary functions at hand so that we arrive at 


$$
\begin{gathered}
\beta_{l}=p\left(B x_{l t}+B_{l}\right)+b x_{l t}+b_{l} \\
\omega_{\jmath K H}=C_{\imath G} \epsilon_{\imath \jmath} \epsilon_{G K H} x_{l t}+C_{\jmath K H} \\
\alpha_{H}=p J a_{H}-\frac{1}{2} \rho J a_{H} x_{\mathfrak{t} t} x_{i t}+\sigma_{H} .
\end{gathered}
$$

with $B, B_{l}, b, b_{l}, C_{i G}, C_{\jmath K H}, a_{H}$, and $\sigma_{H}$ depending on $X_{H}, t$, and $x_{i} ; C_{J_{K}}=-C_{\jmath_{H} K}$. An obvious integration provides

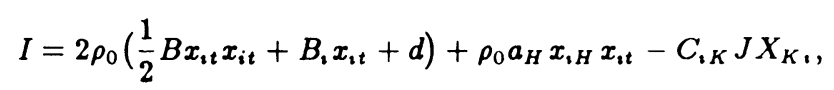

with $d=d\left(X_{H}, t, x_{\imath}\right)$, whereas the expression for $I_{H}$ becomes

$$
\begin{aligned}
I_{H}= & {\left[p\left(B x_{l t}+B_{l}\right)+b x_{l t}+b_{l}\right] 2 J X_{H l} } \\
& +\left(C_{\imath G} \epsilon_{i j l} \epsilon_{G K H} x_{l t}+C_{j K H}\right) x_{\jmath K}+p J a_{H}-\frac{1}{2} \rho_{0} a_{H} x_{l t} x_{l t}+\sigma_{H} .
\end{aligned}
$$

Further information on $I$ and $I_{H}$ follows from the analysis of (4.6). Substitution for $I$ and $I_{H}$ from (4.15) and (4.16) into (4.6) yields

$$
\begin{aligned}
& p J\left[\left(\frac{\partial B}{\partial X_{H}} x_{i t}+\frac{\partial B_{l}}{\partial X_{H}}\right) 2 X_{H t}+\left(\frac{\partial B}{\partial x_{i}} x_{i t}+\frac{\partial B_{i}}{\partial x_{i}}\right) 2+\frac{\partial a_{H}}{\partial X_{H}}+\frac{\partial a_{H}}{\partial x_{i}} x_{i H}\right] \\
& +\frac{1}{2} \rho_{0}\left[2 \frac{\partial B}{\partial x_{i}} x_{i t}-\left(\frac{\partial a_{H}}{\partial X_{H}}+\frac{\partial a_{H}}{\partial x_{i}} x_{i H}\right)\right] x_{h t} x_{h t} \\
& +\rho_{0}\left(\frac{\partial B}{\partial t} \delta_{i h}+2 \frac{\partial B_{h}}{\partial x_{i}}+\frac{\partial a_{H}}{\partial x_{i}} x_{h H}\right) x_{i t} x_{h t} \\
& +\left[\left(-\frac{1}{2} \frac{\partial C_{r S}}{\partial x_{i}} x_{i t}+\frac{\partial b}{\partial X_{S}} x_{r t}\right) x_{b B}+\frac{\partial C_{r S}}{\partial x_{i}} x_{b t} x_{i B}\right] \epsilon_{r j b} \epsilon_{S R B} x_{j} R \\
& +\left(\rho_{0} \frac{\partial a_{H}}{\partial t} \delta_{i j}+\frac{\partial C_{r S}}{\partial X_{K}} \epsilon_{r j i} \epsilon_{S H K}\right) x_{i t} x_{j H}+\left(\frac{\partial C_{j K H}}{\partial X_{H}}+\frac{\partial \sigma_{K}}{\partial x_{j}}\right) x_{j} K \\
& +\left[\left(-\frac{1}{2} \frac{\partial C_{r} S}{\partial t}+\frac{\partial b_{r}}{\partial X_{S}}\right) \epsilon_{r j b} \epsilon_{S R B}+\frac{\partial C_{j R B}}{\partial x_{b}}\right] x_{j_{R} x_{b B}} \\
& +\left[2 \rho_{0}\left(\frac{\partial B_{i}}{\partial t}+\frac{\partial d}{\partial x_{i}}\right)+2 J \frac{\partial b}{\partial x_{i}}\right] x_{i t}+2 \rho_{0} \frac{\partial d}{\partial t}+\frac{\partial \sigma_{H}}{\partial X_{H}}+2 J \frac{\partial b_{i}}{\partial x_{i}}=0 .
\end{aligned}
$$

The vanishing of the overall contribution of the terms linear in $p$ and terms of second and higherorder in the components $x_{i t}$ leads to

$$
\begin{gathered}
B=\text { constant, } \\
B_{\mathfrak{i}}=\omega_{i k}(t) x_{k}+m_{\mathfrak{i}}(t), \quad \omega_{i k}=-\omega_{k i} \\
a_{H}=a_{H}\left(X_{K}, t\right), \quad \frac{\partial a_{H}}{\partial X_{K}}=0 .
\end{gathered}
$$

Then the vanishing of the terms in the products $x_{t t} x_{R}$ provides

$$
\frac{\partial a_{H}}{\partial t}=0, \quad b=b\left(x_{i}, t\right), \quad C_{1, K}=C_{2, K}(t) .
$$

Further, the vanishing of the coefficient of $x_{i t}$ yields

$$
\frac{\partial}{\partial x_{\mathrm{v}}}\left(2 \rho_{0} d+2 J b\right)=-2 \rho_{0} \frac{\partial B_{\mathrm{i}}}{\partial t} .
$$

Taking the $\partial / \partial x$, derivative of $(4.20)$ and requiring the symmetry in $i$ and $j$ of the resulting expression we find, in view of (4.18), 


$$
\omega_{i k}=\text { const. }
$$

Equation (4.20) can now be solved to give

$$
2 \rho_{0} d=-2 J b-2 \rho_{0} \dot{m}_{i} x_{i}+\alpha(\mathbf{X}, t) .
$$

We now observe that $b$ and $\alpha$ may always be represented as

$$
b=\frac{\partial \nu_{1}(\mathbf{x}, t)}{\partial x_{i}}, \quad \alpha=\frac{\mu_{H}(\mathbf{X}, t)}{\partial X_{H}}
$$

then (4.21) can be given the form

$$
2 \rho_{0} d=D_{H}\left(-2 \nu_{i} J X_{H i}-2 \rho \dot{m}_{\imath} x_{h} x_{h} J X_{H_{\imath}}+\mu_{H}\right)
$$

thus showing that the contribution of $d$ to the conserved density (4.15) is represented in the form of a divergence and consequently may be disregarded, independently of the explicit form of $\nu, \mu$, and $\dot{m}_{i}$, that is of $b$ and $\alpha$. Similarly, we have

$$
-C_{i K} J X_{K \imath}=D_{H}\left(-\frac{1}{2} C_{i K} \epsilon_{i j h} \epsilon_{K A H} x_{j A} x_{h}\right),
$$

where the condition $C_{i K}=C_{i K}(t)$ has been taken into account, whence it follows that the contribution of $C_{i K}$ to the conserved density can be disregarded as well.

As a consequence, the explicit determination of the unknowns $b, \alpha, C_{i K}, C_{K_{K}}, \sigma_{H}$, and $b_{l}$ is not required, since we are allowed to consider an equivalent conservation law where the expression of the density does not involve $d$ and $C_{i K}$; at most the preceeding unknowns can influence the expression of the flux, and no essential information is lost if we set them equal to zero.

In conclusion, we can write the set of conservation laws through the 4-tuple $\left(I, I_{H}\right)$ as

$$
\begin{gathered}
I=2 \rho_{0}\left(\frac{1}{2} B x_{h t} x_{h t}+x_{h t} \omega_{h k} x_{k}+m_{h}(t) x_{h t}\right)+\rho_{0} a_{H}(\mathbf{X}) x_{\imath t} x_{i H}, \\
I_{H}=p\left[B x_{l t}+\omega_{l k} x_{k}+m_{l}(t)\right] 2 J X_{H l}+a_{H}(\mathbf{X})\left(p-\frac{1}{2} \rho x_{l t} x_{l t}\right) J
\end{gathered}
$$

$a_{H}$ being a divergence-free vector.

\section{EXPLICIT FORM OF THE CONSERVATION LAWS.}

The direct approach of the previous section has provided an exhaustive set of independent conservation laws for incompressible fluid motions. To discuss the physical interpretation of the results we observe that the arbitrariness of $m_{h}, \omega_{h k}$, and $B$ leads to the conservation laws for the linear momentum (in a generalized form), the angular momentum, and the energy, respectively. Indeed, the law for linear momentum derived here, where each conserved component - say $\rho_{0} x_{h t}$ - is multiplied by an arbitrary function of the time $m_{h}(t)$, constitutes an extension of the usual formulation which corresponds to $m_{h}$ being constant. As a direct calculation can show, the greater generality is due to the incompressibility constraint; this interpretation is further enforced by the observation that a similar result also holds for incompressible viscous fluids [8].

As to $a_{H}$, we have

$$
I=\rho_{0} a_{H} v_{i} x_{i}, \quad I_{H}=a_{H}\left(p-\frac{1}{2} \rho v^{2}\right) J
$$


The law (5.1) mirrors the constraint of mass conservation for the coordinate transformation in the reference configuration $\mathcal{F}$, as follows from a comparison with the results obtained in [9].

Each of the above conservation laws has a counterpart holding for compressible fluids, with the only exception of the conservation of linear momentum which requires a constant $m_{h}$ [9]. In this connection it is rather unexpected that no center-of-mass theorem has been found, although it has been shown to hold for compressible fluids $[8,9]$ as a continuum counterpart of a relation derived by Hill [12] for a system of $N$ particles. In the present case this result is a consequence of the conservation law of linear momentum. In fact, it suffices to set $m_{h}(t)=\bar{m}_{h} t / 2$, with $\bar{m}_{h}$ constant, and to let the remaining arbitrary functions and constants vanish. Then the expression of the conserved density is $I=\rho_{0} \bar{m}_{h} x_{h t} t$ and may be trivially modified by addition of a divergence term of the form $D_{H}\left(-\bar{m}_{i} x_{h} x_{h} \rho J X_{H i} / 2\right)=-\rho_{0} \bar{m}_{h} x_{h}$. The resulting expression of the conserved density turns out to be given by

$$
I=\bar{m}_{h} \rho_{0}\left(x_{h t} t-x_{h}\right)
$$

thus yielding the motion of the center-of-mass [9].

Now we are ready for the final step toward the formulation of conservation laws in the Eulerian description. To this aim we observe that the densities in the Eulerian and the Lagrangian descriptions are related through multiplication by the factor $J$, as follows from the change of variable theorem within multiple integrals [13]. We also make use of the correspondence between material and spatial descriptions of vector fields, $Y_{H} \leftrightarrow y_{h}$, namely [13]

$$
Y_{H}=J y_{h} X_{H h}
$$

and we observe that $D_{H} Y_{H}=J D_{h} y_{h}[13]$, where $D_{h}$ denotes the total derivative with respect to $x_{h}$. Then, on examining separately the various contributions to the general form of the conservation laws in the Lagrangian formulation, we are led to the following independent contributions in the Eulerian description:

$$
\begin{gathered}
D_{t}\left(\rho m_{h} v_{h}\right)+D_{h}\left(\rho m, v, v_{h}+m_{h} p-\dot{m}, x, v_{h}\right) \doteq 0, \\
D_{t}\left(\rho \omega_{h k} v_{h} x_{k}\right)+D_{h}\left(\rho \omega_{j k} v_{j} x_{k} v_{h}+\omega_{h k} p x_{k}\right) \doteq 0, \quad \omega_{h k}=-\omega_{k h}, \\
D_{t}\left(\frac{1}{2} \rho v^{2}\right)+D_{h}\left(\frac{1}{2} \rho v^{2} v_{h}+p v_{h}\right) \doteq 0,
\end{gathered}
$$

where constant factors have not been considered and the contributions to the conserved flux without analogue in in the expression for $I_{H}$ are due to the fact that we consider fixed regions in the physical space. It has already been pointed out that eq.(5.2) generalizes the usual conservation law of linear momentum, $m_{h}$ being an arbitrary function of time. Equations (5.3) and (5.4) are the usual conservation laws of angular momentum and energy. In this connection the "nter " ${ }^{-}$-inass theorem takes the form

$$
D_{t}\left[\rho\left(x_{k}-v_{k} t\right)\right]-D_{h}\left[\rho\left(x_{k}-v_{k} t\right) v_{h}-t p \delta_{k h}\right] \doteq 0,
$$

and may be regarded as equivalent to the conservation law (5.2).

To find the analogue of the conservation law determined by (5.1) it is appropriate to introduce the Eulerian counterpart of $J a_{H}$, say $a_{i}$, which is given by $a_{\mathfrak{i}}=a_{H} x_{i}$. Accordingly, we find $I=\rho a_{i} v_{i}$ and $I_{H}=\left(p-\frac{1}{2} v^{2}\right) J a_{l} X_{H l}$ and hence the Eulerian form of the conservation law reads 


$$
D_{t}\left(\rho a_{1} v_{i}\right)+D_{h}\left[\rho a_{i} v_{i} v_{h}+\left(p-\frac{1}{2} v^{2}\right) a_{h}\right] \doteq 0 .
$$

The conserved density is just the projection of the linear momentum on the field $a_{i}$. The vector $a_{i}$ is divergence free, since $a_{H}$ is, and satisfies the further condition

$$
\frac{\partial a_{i}}{\partial t}+\frac{\partial a_{i}}{\partial x_{h}} v_{h}-a_{h} \frac{\partial v_{i}}{\partial x_{h}}=0
$$

which is the Eulerian correspondent of the condition that $a_{H}$ is independent of $t$. In practical terms, however, it is easily seen that within the usual Eulerian framework eq.(5.7) admits only the vanishing solution. This shows that the conservation law (5.6) can only be arrived at within the Lagrangian formulation and explains why Olver [5] could not find it.

\section{ACKNOWLEDGEMENTS.}

The paper has been partially supported by the National Group for Mathematical Physics of the Italian Research Council and by the Italian Ministry of the Public Education through the research project "Problemi di evoluzione nei fluidi e nei solidi".

\section{REFERENCES}

1. SERRE, D. Les invariants du premier ordre de l'equation d'Euler en dimension 3, C. R. Acad. Sc. Paris 289 (1979) 267-270.

2. STRAUSS, W. A. Nonlinear invariant wave equations, in Invariant Wave Equations, G. Velo and A. S. Wightman Eds., Springer-Verlag, New York, 1978, 197-249.

3. EBIN, D. G. Integrability of perfect fluid motion, Commun. Pure Appl. Math. 36 (1983) 37-54.

4. MOFFATT, H. K. The degree of knottedness of tangled vortex lines, J. Fluid Mech. 35 (1969) 117-129.

5. OLVER, P. J. Olver A nonlinear Hamiltonian structure for the Euler equations, J. Math. Anal. Appl. 89 (1982) 233-250.

6. CAVIGLIA, G. Symmetry transformations, isovectors and conservation laws, J. Math. Phys. 27 (1986) 972-978.

7. BENATI, M. and CAVIGLIA G. Conservation laws for 3-dimensional compressible Euler equations, Int. J. Engng. Sci. 25 (1987) 1-7.

8. CAVIGLIA, G. Composite variational principles and the determination of conservation laws, J. Math. Phys., in print.

9. CAVIGLIA, G. and MORRO, A. Noether-type conservation laws for perfect fluid motions, J. Math. Phys. 28 (1987) 1056-1060.

10. OLVER, P. J. Applications of Lie Goups to Differential Equations, Springer-Verlag, New York, 1986.

11. GURTIN, M. E. An Introduction to Continuum Mechanics, Academic Press, New York, 1981.

12. HILL, E. L. Hamilton's principle and the conservation theorems of mathematical physics, Rev. Mod. Phys. 23 (1951) 253-260.

13. MARSDEN, J. E. and HUGHES, J. R. Mathematical Foundations of Elasticity, Englewood Cliffs, N. J., 1983. 


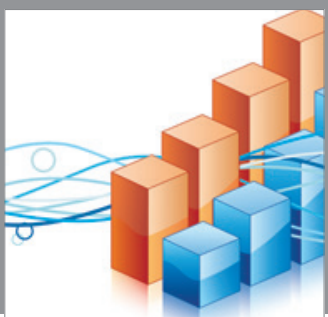

Advances in

Operations Research

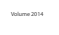

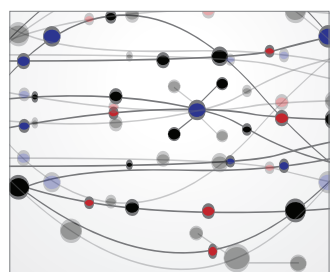

\section{The Scientific} World Journal
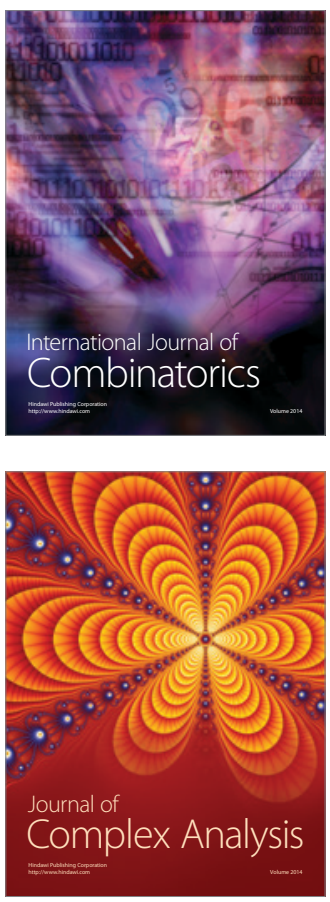

International Journal of

Mathematics and

Mathematical

Sciences
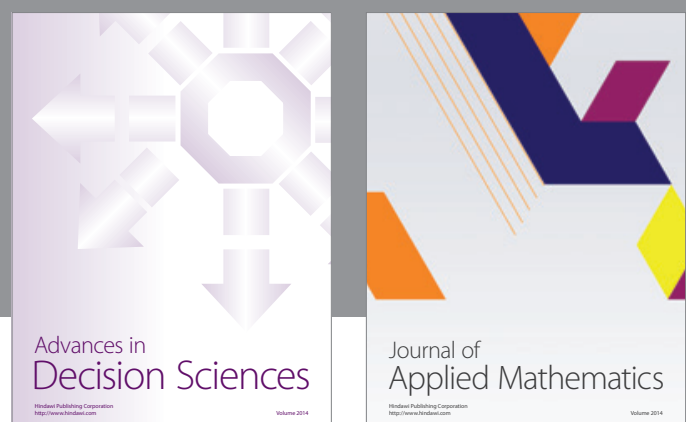

Journal of

Applied Mathematics
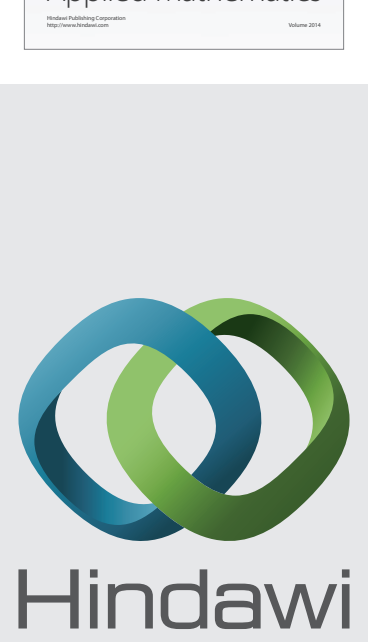

Submit your manuscripts at http://www.hindawi.com
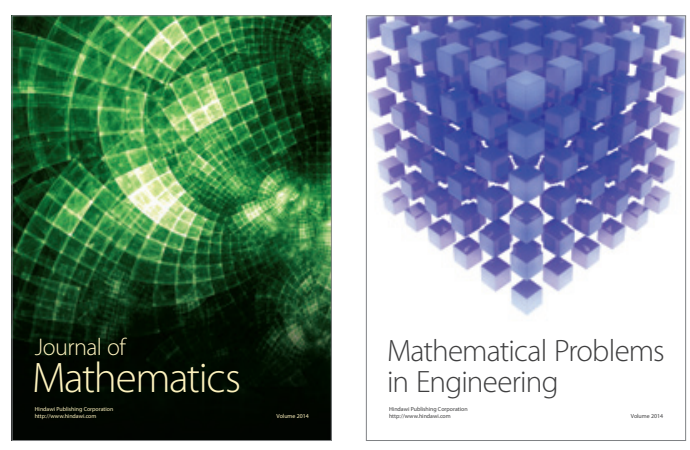

Mathematical Problems in Engineering
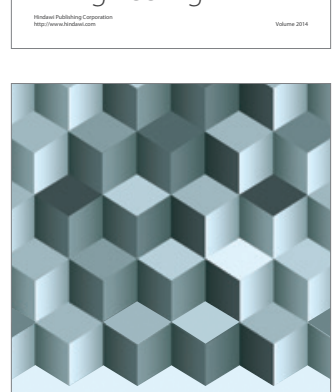

Journal of

Function Spaces
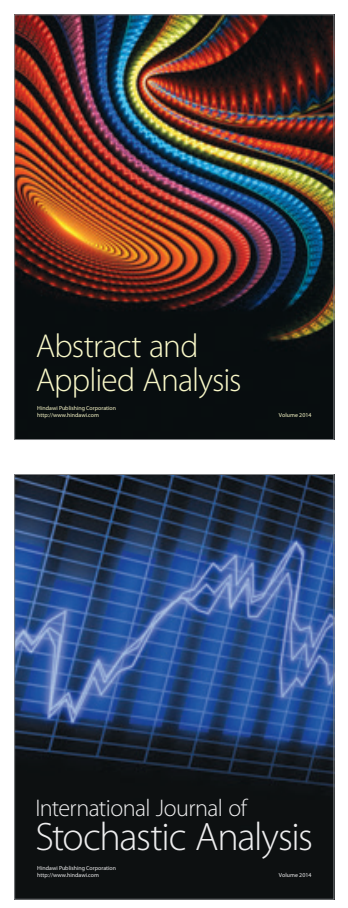

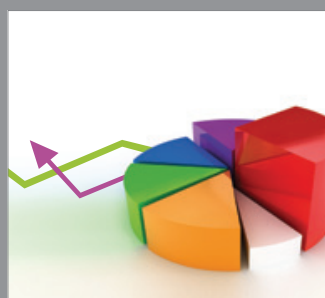

ournal of

Probability and Statistics

Promensencen
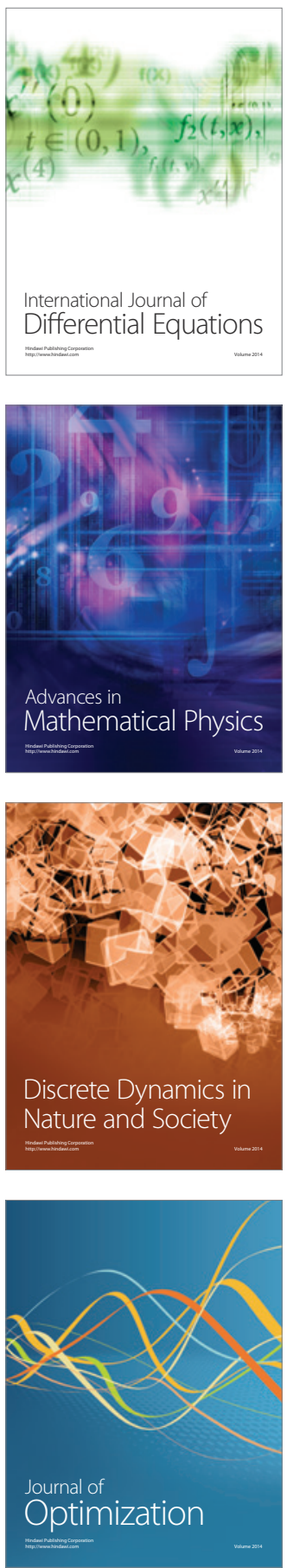